\title{
Numerical Investigation of the Effect of Tread Pattern on Rotating Wheel Aerodynamics
}

\author{
M.Z. Soliman'1 A.R. El-Baz², M.A. Abdel-Aziz'1 , N. Abdel-Aziz³ and O.S. Gabor ${ }^{4}$ \\ ${ }^{1}$ Automotive Engineering, Ain-Sham University, Cairo, Egypt \\ Phone: +201090698853 \\ ${ }^{2}$ Renewable Energy Department, British University in Egypt, Cairo, Egypt \\ ${ }^{3}$ Mechanical Power Engineering, Ain-Sham University, Cairo, Egypt \\ ${ }^{4}$ Aeronautical Engineering, University of Salford, UK
}

\begin{abstract}
The present work investigates the dynamic effect of wheel rotation on the aerodynamic characteristics of slick type (ST) wheel of Formula One racing cars using a computational approach. The ST wheel model was compared to experimental results as a validation case. The pressure coefficient over the ST wheel circumference at its middle plane (xy) has been considered as an experimental case from literature and the computed results showed a reasonable agreement. Furthermore, a quantitative evaluation of the numerically-determined wheel drag, local separation and stagnation angles has been also compared to those extracted experimentally from literature. The validation work served by assessing the suitability of using Moving Reference Frame (MRF) method to simulate the effect of wheel rotation, as well as defining the most reliable conditions of testing such as the optimal meshing criteria, the computational domain size, and the adopted turbulence model. According to wheel studies, all computational work was performed at a Reynolds number of $6.8 \times 10^{5}$ based on the wheel diameter. The wheels aerodynamic drag, lift, and moment coefficients were computed for each wheel model. Further parametric study on the tread design of the tread type (TT) wheel was performed by varying the tread depth, h. Besides, general schematic pictures of the flow behavior around the TT wheel are presented.
\end{abstract}

ARTICLE HISTORY

Received: $3^{\text {rd }}$ Apr 2020

Revised: $8^{\text {th }}$ Oct 2020

Accepted: $13^{\text {th }}$ Nov 2020

\section{KEYWORDS}

Automotive aerodynamics; Wheel aerodynamics;

Tire CFD;

Rotating wheel dynamics

\section{INTRODUCTION}

According to modern aerodynamics perspective, body shape optimisation becomes less influential on producing significant improvements in overall vehicles aerodynamic performance. Wheel aerodynamics recently becomes an interesting research area, especially for the racing cars since the wheel is entirely exposed to the oncoming airflow. Many researchers have oriented their attention on studying the dynamic effect of wheel rotation on the vehicle aerodynamics. Measuring the aerodynamic forces acting on the fully exposed rotating wheel in contact with the ground was started by Morelli [1] using the direct experimental method. His attempt was based on simulating the wheel deformation by putting it into a rectangular ground recess with an opened gap of $0.016 \mathrm{D}$, where $\mathrm{D}$ is the wheel diameter. It has been found that the effect of rotation produces an increase in the drag by $10 \%$ compared to that of stationary wheel and rotating wheel in ground contact would produce a net downforce; where $\mathrm{Cl}=-0.075$.

Further attempts followed Morelli [1] to simulate the wheel ground contact, one being done by Stapleford and Carr [2]. Their experimental test was performed using paper strips to seal the wheel ground clearance. It has been concluded that the wheel rotation in contact with stationary ground generated a significant lift force; where $\mathrm{Cl}=0.4$. This result came in contrary to Morelli's conclusion [1]. They recommended that the correct way to simulate the rotating wheel could be reached only by using real wheel ground contact, but the moving ground has not a considerable effect on the outcomes. Cogotti [3] tried to build a variable wheel height mechanism to study the effect of the rotating wheel approaching the ground and verify the previously mentioned contradicting conclusions. Based on the obtained results at the possible minimum gap, his conclusion emphasised those of Stapleford and Carr [2]. He confirmed that Morelli's [1] lift force results having some faults in measurements. Thus, a wheel rotating in ground contact always produces a lift force.

The first who approached the reality of wheel ground contact were Fackrell and Harvey [4, 5]. The wheel was placed on a moving belt, and the net acting forces were computed using a set of on-surface pressure sensors. Their results confirmed, in a qualitative agreement with what was concluded by Stapleford and Carr [2] and Cogotti [3], that the effect of ground contact produces significant lift. Also, the results indicated a higher lift coefficient and lower drag coefficient for the wheel, which has a more rounded profile. They also captured a pressure peak $(\mathrm{Cp}>2)$ at the head of the contact patch caused by the dynamic effect of wheel rotation and proposed that another suction peak should be located at the back, but they couldn't capture it due to the moving belt lift behind the wheel. Hinson [6] followed the same attempt of 
Fackrell and Harvey [4, 5] using more advanced pressure measuring techniques and her results were a very good agreement to previous data.

Further relevant researches were done by Mears [7, 8] to experimentally investigate the aerodynamic forces on a pneumatic go-kart wheel using an improved measurement system. This new system made a novel contribution in recording the suction peak at the back of the contact patch as proposed by Fackrell and Harvey [4, 5]. Purvis [9] measured the wake structure behind the rotating wheel, and two-lobe vortices were observed near the contact patch. His experimental setup was performed at different vertical loads to vary the wheel deformation and side profile. It was found that the lobe size increases dramatically with increases in the vertical load.

Van Den Berg [10] started to include the front wings to the wheel setup to experimentally study the aerodynamic interaction between them using advanced flow visualisation methods. He was the first who used Particle Image Velocimetry (PIV) technique, and his results led to a breakthrough of capturing a re-attaching flow behaviour over the wheel surface behind the separation region. Van Den Berg also attempted to simulate the wheel model computationally using various turbulence models. He concluded that no significant deviations were detected between realisable k- $\varepsilon$, $k-\omega$ SST or DES models in predicting the surface pressure distribution and aerodynamic coefficients. Knowles [11] attempted to investigate the flow characteristics around the wheel using Laser Doppler Anemometry (LDA). Three velocity components were measured across four planes downstream to capture the trailing vortices. It has been concluded that an inverted-T vortex shape, a pair at the upper and another separated wider one near the ground, was found behind the rotating wheel. A merged interaction between the upper and ground vortex pairs occurred within a distance of wheel diameter D.

Heyder-Bruckner [12] continued on the work done by Van Den Berg [10] using the same experimental setup. A more detailed transient study was dedicated to analysing the flow separation mechanism that was observed by Van Den Berg [10]. He concluded that the instantaneous flow at the separation region creates periodic shed vortices downstream which lead to drag and lift fluctuations. Sprot [13] experimentally investigated the flow field around the rotating wheel concerning the fact that the shape of rounded side profile has a significant effect on the pressure distribution results as concluded by Fackrell and Harvey $[4,5]$. Tests were performed at various wheel inflation pressures and heights using a typical deformable wheel. His experimental arrangement was fitted with PIV for flow visualisation and load-cell for forces measurements. It was found that the wheel sidewall shape produces significant changes to the flow field downstream and is much more than that of the contact patch area.

According to the authors' knowledge, the first to use the computational approach to simulate the rotating wheel aerodynamics was Axon [14] in 1998. His work was aiming eventually on verifying a reliable computational wheel model using a simplified shape of Fackrell's wheel geometry. Compared to Fackrell's experimental outcomes, the obtained results were successfully capturing the pressure distribution curve over the wheel surface as well as capturing the suction peak. He also concluded that an optimum solver configuration was developed which comprised of the realisable k- $\varepsilon$ turbulence model with full boundary layer closure. Skea [15] attempted to use the symmetry condition to define the ground surface to build a more economical model. However, his assumption did not differ a lot in the computed results from that obtained by Fackrell, but he recommended on simulating the ground as a moving wall to be the correct approach.

McManus' [16] work aimed at revisiting all the inaccurately modelled geometries and to avoid the simplifications. He modelled Fackrell and Harvey's exact geometry with the proper side profile and without using rim covers. He simulated the flow using RANS, URANS and DES. His RANS and URANS results were promising, showing good agreement. He also concluded that DES results were the best in obtaining accurate predicted wakes although similar pressure distributions and force coefficients were predicted using RANS and URANS models. That was also emphasised by Basara et al. [18] who used a steady RANS model in predicting the wheel aerodynamic coefficients. WÄaschle [17] applied an unsteady RANS solver and presented averaged results following Basara et al. [17] trend. His study came contrary to that of Basara et al. [17]. He stated that unsteady solver modelling predicts the force coefficients better than the steady. He used realisable k- $\varepsilon$ URANS simulation, which showed that the difference in lift and drag values are $1 \%$ and $5 \%$ from the experimental ones respectively. He concluded that the lack of inaccurate prediction of the lift coefficient was due to a $10^{\circ}$ earlier-predicted separation at the top of the wheel.

Diasinos et al. [19] utilised a CFD model for the wheel ground assembly aiming at investigating the influence of wheel hub geometry on the wakes and vortices regions. Three models of flattened wheel sides, curved side wall and simple rim features were considered in this study. He concluded that the detailed rim has a significant influence on the wake structure and can produce variations in the $\mathrm{Cd}$ and $\mathrm{Cl}$ by around $8 \%$ and $30 \%$ respectively.

Soliman et al. [20] computed the aerodynamic moment coefficient of the wheel model for ST and TT wheel types using realisable k- $\varepsilon$ URANS. His work aimed at evaluating this previously undetermined parameter. It found that the values of moment coefficient were approximately -0.015 and -0.09 for ST and TT wheels model respectively. The negative sign indicates that the moment applied is resistive to the wheel motion. He concluded that the moment coefficient has a negligible effect on the overall ST wheel performance while, for the TT, a considerable effect is obtained since the developed resistive torque is nearly four times that of the typical wheel rolling resistance. With regards to including the influence of wheel tread pattern, the main problem is concerning with the rotating tread pads which need a kind of sliding mesh to consider its effect numerically. This was the main challenging point in this new considered feature because the sliding mesh approach has an extremely high computational cost, especially for three-dimensional models. Several trials have been done, such as considering the tread features as a rough surface in the modelling. This approach was adopted in Schnepf et al. [21] work for the sake of simplicity. He concluded that it would be more accurate and realistic if the detailed tread geometry is included. Another attempt to investigate the reliability of using moving reference frame approach, that 
was used by Soliman et al. [20], in comparison with a full sliding mesh, the model was carried out by Hobeika and Sebben [22]. They concluded that moving reference frame is a good promising approach for simulating the wheel tread influences on the flow for low computational cost.

Bolzon et al. [23] investigated the effect of wheel configurations experimentally and shape aspects on the overall drag of a $1 / 5^{\text {th }}$ scale passenger vehicle. Amongst his important conclusions, he stated that as the rim coverage area decreases, the local wheel drag increases. In addition, the tread pattern has no significance on the local wheel drag coefficient when wheels were stationary. However, it increases by 2 counts once they became rotating.

\section{OBJECTIVE}

According to the reviewed literature, most wheel aerodynamic studies focused on predicting the force coefficients and the flow features around it. The drag and lift forces are not the only dominating parameters in the evaluation of the rotating wheel aerodynamics. An additional factor should be included and could be considered as a resistive moment to the wheel rotation. This additional resistance has not been determined previously in literature except by Soliman et al. [20], and its effects are still poorly understood. Figure 1 shows a side view of a typical isolated rotating wheel in contact with the ground. The figure also demonstrates the effort that the wheel has to exert to keep its rotation against the upcoming flow streams since it is fully exposed.

The additional resistive moment is considered as a new parameter, namely resistive torque, which in turn tends to stop the wheel due to the vicious action in case of ST wheel since the wheel surface is smooth. On the other hand, for TT wheel, the wheel surface has tread pads and patterns in its shape. The tread pads could represent an obstacle to the accelerating flow over the upper wheel surface; hence the resistive torque is expected to be higher and becomes a more influential factor. The present work is concerned with studying the dynamic effect of wheel rotation on the generated aerodynamic forces and moment of the ST and TT condition wheels. Also, a considerable effort is directed to take the tread pattern into account since the value of the resistive torque is expected to be strongly dependent on it.

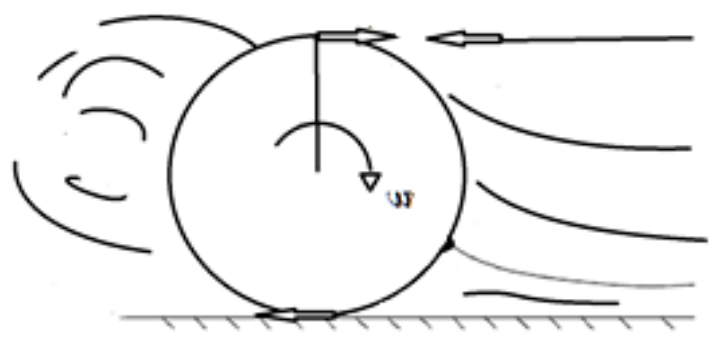

Figure 1. Schematic diagram of a rotating wheel moving against the flow direction [20].

\section{WHEEL MODEL}

\section{ST Wheel Geometry}

A three-dimensional model of half-scale ST racing wheel is developed based on the experimental Van Den Berg's [10] wheel geometry. Figure 2 shows the wheel geometrical shape and the detailed dimensions as well. Four longitudinal grooves are created and distributed with equal spacing over the wheel flattened surface, and its rounded corners are considered. The wheel contact patch region is developed using Plint technique to ensure satisfied grid quality by eliminating the severely-sharp profile and avoid excessive cell stretching [16].

\section{TT wheel geometry}

The TT wheel is created with a half-scale model. The wheel model's shape and dimensions were created in accordance with the typical Bridgestone Potenza Formula One tire specifications (20×7.5-13) [24]. The wheel's main dimensions and other details related to the tread pattern are depicted in Figure 3. The typical tread pads depth, h, is $3 \mathrm{~mm}$ and its width is $20.16 \mathrm{~mm}$ arranged with $7.5 \mathrm{~mm}$ spacing. The same plint technique is also developed on the TT wheel model. Some real details of the tread pattern are trimmed in order to simplify the model; every extremely adjacent tread pads are merged, the tread pads were modified to be rectangular and equally spaced instead of being rhombus with various spacing. As a result, each tread rib has mainly five tread pads and is flattened to avoid the complicity of V-curved tread shape.

\section{Computational Domain}

The dimensions of the computational domain are set according to the compromise between the recommended dimensional ratios in previous studies $[14,16,17]$ and the experimental test section size. Figure 4 shows the overall dimensions that are adopted for the two wheels cases. The upstream length ahead the wheel is 6D. The right and left sides, and top plane lengths are set to be $3 \mathrm{D}, 3 \mathrm{D}$ and $6 \mathrm{D}$ far away from the wheel model respectively. The downstream length 
behind the wheel is $15 \mathrm{D}$ to provide enough space for monitoring the high developed turbulence there since the wheel represents as a bluff body. After all, the computational domain has an approximately blockage ratio equals to $6.2 \%$.

The flow domain is split into two main regions to develop a mesh with different grid sizes. The first region is a rectangular shape surrounding the wheel and is extended gradually with wider width until it reaches 8D downstream, as shown in Figure 4. The remaining flow domain is the second region which is dominated by the free-stream flow. Also, based on the symmetrical shape of the studied model, the only half domain size is considered. The total flow domain is bisected; hence, the number of elements decreases to half, and the computational time decreased. Further circular splitting to the flow domain around the wheel is created by an offset of $0.5 \mathrm{~h}$ from the wheel surface in order of enabling a rotating zone, where $\mathrm{h}$ is the tread depth. This is a new concept of representing the rotating flow region near the wheel's wall. This concept is necessary when the tread pattern exists, in order to simulate the fan effect, which interacts with the upstream flow over the rotating wheel. Moving Reference Frame MRF technique is applied in this region [25].

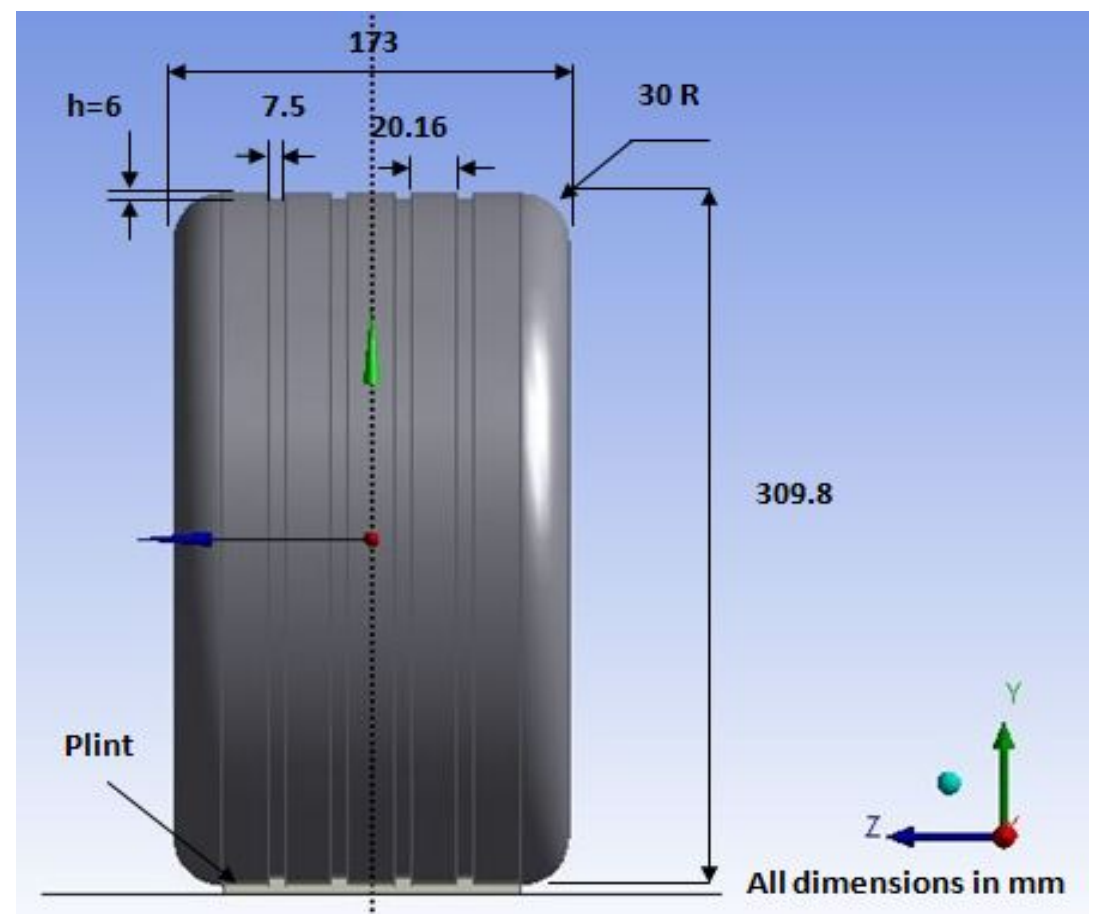

Figure 2. The ST wheel geometry $[10,20]$.

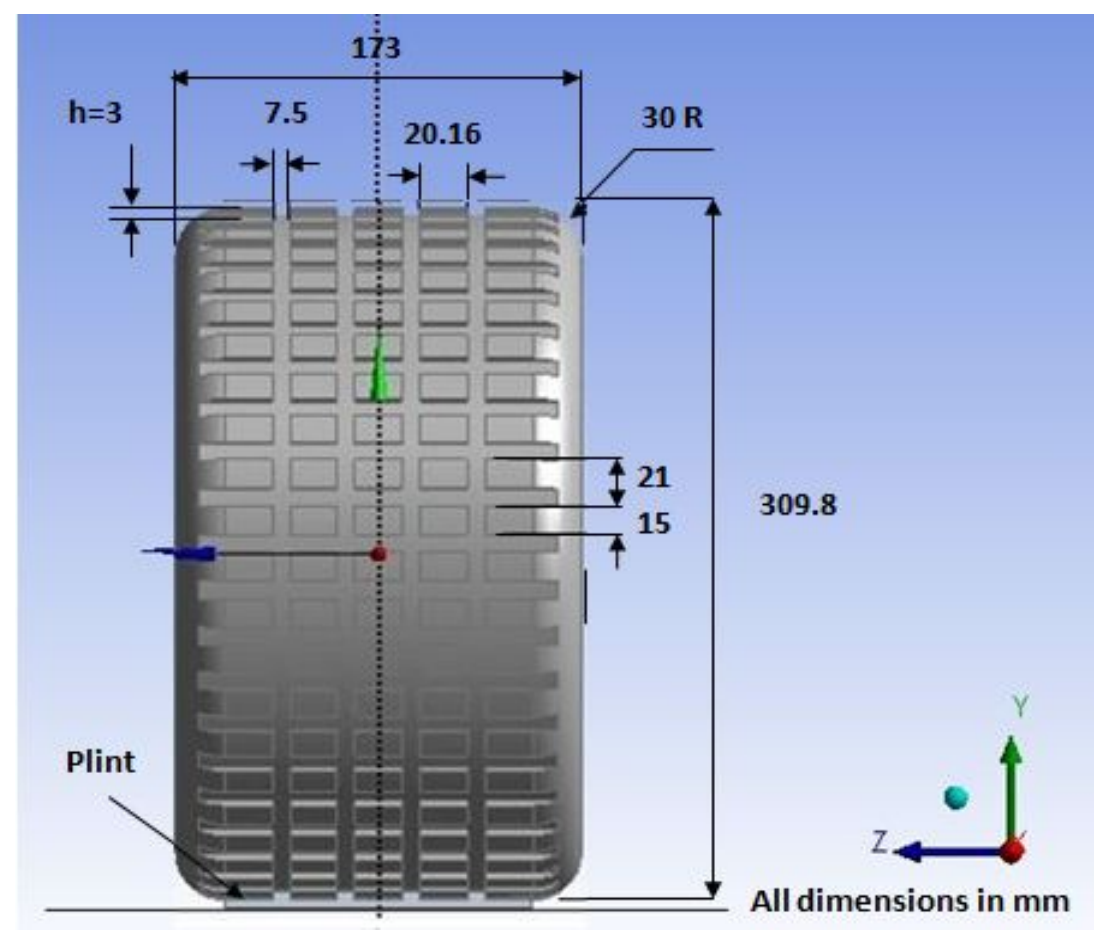

Figure 3. The TT wheel and its tread pattern. 


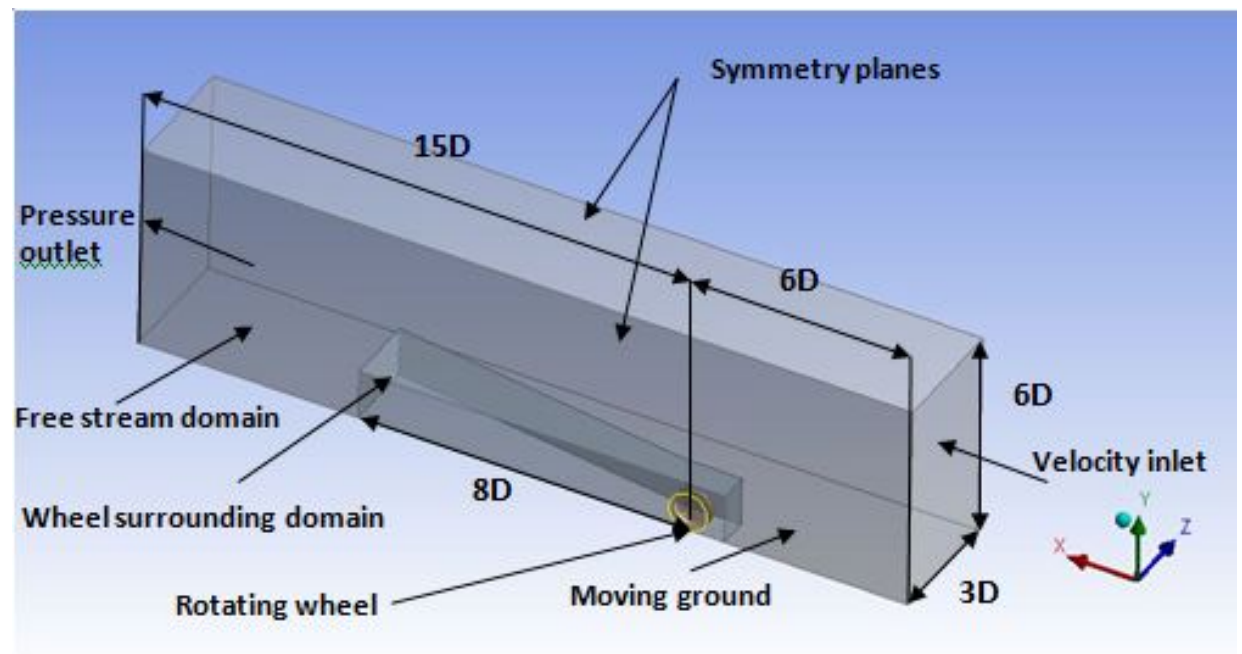

Figure 4. Schematic diagram of the computational domain showing the splitting of volume around the wheel body and the selected dimensions as well as the utilised boundaries condition.

\section{BOUNDARY CONDITIONS}

In order to correctly define the model boundaries, it should be close as possible to the real conditions. As shown in Figure 4, the flow domain boundaries conditions were defined as; velocity inlet to the inlet boundary, with a velocity magnitude equal to $30 \mathrm{~m} / \mathrm{s}\left(\operatorname{Re}=6.8 \times 10^{5}\right.$ based on wheel diameter), pressure outlet condition to the outlet boundary, and a smooth $30 \mathrm{~m} / \mathrm{s}$ moving wall with a no-slip condition to the ground surfaces. Both side and top boundaries are assumed to be symmetry planes. This assumption was set to omit excessive grid refinement there. The wheel surface was defined as a smooth wall without slipping condition with a rotational speed $\omega=193.7 \mathrm{rad} / \mathrm{s}$ about its centre. The upstream flow turbulence intensity is defined as $0.3 \%$ as experimentally obtained [25].

\section{MESH GENERATION}

In the present work, a meshing strategy was applied to minimise the number of elements in the free stream regions and maximising those in downstream and near-wall regions to provide an optimised model. The meshing strategy was utilised based on the previously mentioned domain splits. Furthermore, near-wall treatment is used to predict the flow behaviour over the wheel surface to minimise the total cell count. As shown in Figure 5, the wheel surface Y+ is adjusted to be within the range of $25-300$ which is defined as:

$$
Y+=y \frac{\sqrt{\frac{\tau_{w}}{\rho}}}{v}
$$

where, $y$ is the normal distance from the surface to the first cell centre, $\tau_{w}$ is the surface shear stress and $v$ is the kinematic viscosity. In order to enable surface $\mathrm{Y}+$ being within the optimum range, $y$ is adjusted to $2 \mathrm{~mm}$. Based on the available computational resources, the total number of elements was manipulated over the flow domain to make sure it does not exceed the maximum limit of 7 million cells.

Hybrid Tetra and Hexahedron grid has been constructed for all domain sections since the geometry complexity level is high, as shown in Figure 6. In addition, several attempts were made to recover the relatively highly-skewed cells to ensure good cell quality, especially near the wheel to a limitation of 0.3 maximum skewness and 2 aspect ratio to provide stable conversions and reliable outcomes [25]. In order to investigate the influence of grid size on the computationally obtained outcomes, six grid sizes were examined. The test was applied over the grid size of the surrounding wheel region while keeping it fixed by $30 \mathrm{~mm}$ over the free-stream region. The test was initiated using $20 \mathrm{~mm}$ followed by $16,12,8$, 5 and $4 \mathrm{~mm}$, resulting in approximately 2,3,4,5,6 and 7 million grid count respectively. It has been found that at 5, 6 and 7 million grid count, mesh independence is reached based on the invariance of the aerodynamic coefficients, as shown in Figure 7. Therefore, in the present work, 6 million grid count was developed over the whole case studies. 


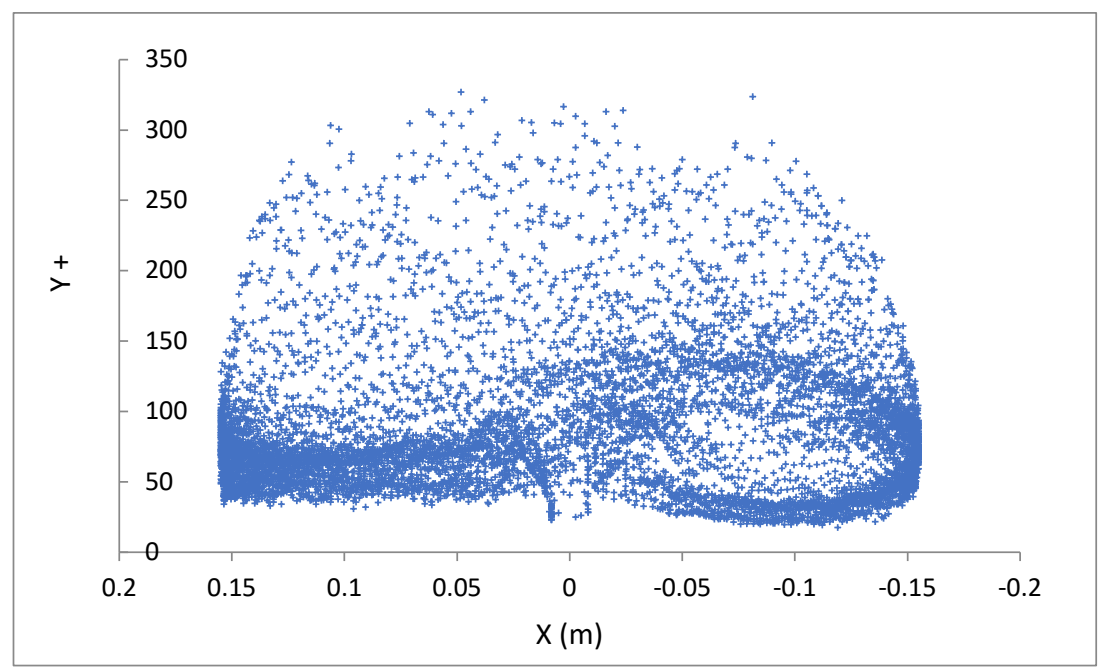

Figure 5. Distribution of $\mathrm{Y}+$ over the TT wheel surface.

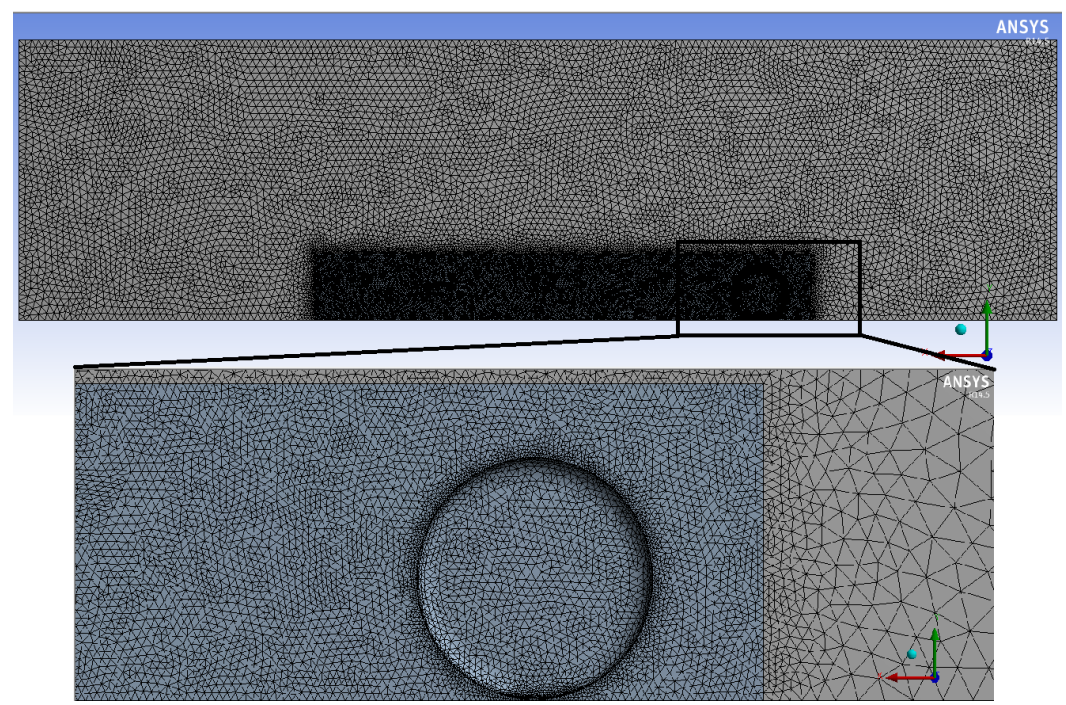

Figure 6. Distribution of mesh in the region around the wheel by $8 \mathrm{~mm}$ grid size and $2 \mathrm{~mm}$ over the wheel surface and showing the unstructured tetrahedral grid.

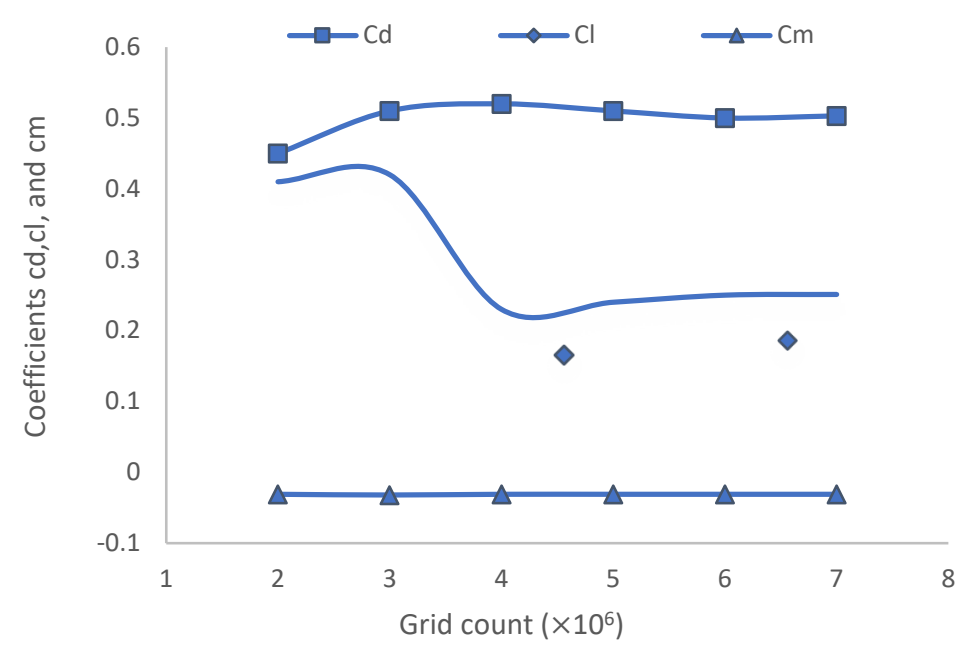

Figure 7. Effect of grid count on the aerodynamic coefficients of ST wheel model.

\section{GOVERNING EQUATIONS AND TURBULENCE MODEL}

Throughout the present work, all cases including the ST and TT wheel have been simulated using Ansys Fluent using segregated solver in implicit form and realisable $\mathrm{k}-\varepsilon$ steady-state RANS. The SIMPLEC scheme for the pressure - 
velocity coupling and an incompressible flow has been selected. The discretisation schemes were set as second-order upwind for the momentum and turbulence scaler quantities. According to the experience gathered from the literature [10, $14,16]$, this solver configuration has been often applied and verified experimentally and showed accurate computational outcomes, especially for deducing the aerodynamic coefficients. However, a validation study has already presented to assess the suitability of using MRF. In the rotating wheel zone, the acceleration of the fluid is augmented by additional terms that appear in the momentum equations and the fluid velocities are transformed from the stationary frame to the rotating frame using the following relation:

$$
\overrightarrow{\mathrm{v}_{r}}=\overrightarrow{\mathrm{v}}-\overrightarrow{u_{r}}
$$

where,

$$
\overrightarrow{u_{r}}=\vec{\omega} \times \vec{r}
$$

In the above, $\overrightarrow{v_{r}}$ is the relative velocity (the velocity viewed from the rotating frame), $\vec{v}$ is the absolute velocity (the velocity viewed from the stationary frame), $\overrightarrow{u_{r}}$ is the 'whirl' velocity (the velocity due to the moving frame), $\omega$ is the rotating frame angular velocity, and $\bar{r}$ is the position vector from the origin of the rotating frame. Consequently, the flow governing mass and momentum conservation equations are set as:

$$
\begin{gathered}
\nabla \cdot(\rho \overline{\mathrm{v}})=0 \\
\partial / \partial t(\rho \overline{\mathrm{u}})+\nabla \cdot(\rho \overline{\mathrm{v}} \overline{\mathrm{v}})=-\nabla p+\nabla \cdot(\overline{\bar{\tau}})+\rho \bar{g}
\end{gathered}
$$

Where $\mathrm{p}$ is the static pressure, $\overline{\bar{\tau}}$ is the stress tensor given by [25]:

$$
\overline{\bar{\tau}}=\mu\left[\left(\nabla \overline{\mathrm{v}}+\nabla \overline{\mathrm{v}}^{T}\right)-\frac{2}{3} \nabla \cdot \overline{\mathrm{v}} I\right]
$$

Where $\mu$ is the molecular viscosity, $I$ is the unit tensor, and the second term on the right-hand side is the effect of volume dilation. For the rest of the domain, the ensemble-averaged mass and momentum equations can be written in the Cartesian tensor form as [25]:

$$
\begin{gathered}
\partial / \partial x_{i}\left(\rho u_{i}\right)=0 \\
\partial / \partial t\left(\rho u_{i}\right)+\partial / \partial x_{j}\left(\rho u_{i} u_{j}\right)=-\partial p / \partial x_{i}+\partial / \partial x_{j}\left[\mu\left(\partial u_{i} / \partial x_{j}+\partial u_{j} / \partial x_{i}-\frac{2}{3} \delta_{i j} \partial u_{i} / \partial x_{l}\right)\right]+\partial / \partial x_{j}\left(-\rho \overline{u_{l}^{\prime} u_{j}^{\prime}}\right)
\end{gathered}
$$

The term $-\rho \overline{u_{\imath}^{\prime} u_{\jmath}^{\prime}}$ in the momentum equation is the Reynolds stresses, which is modelled in order to close Eq. (8). Regarding the convergence criteria, the robust under-relaxation value for pressure was 0.25 , while, for each of momentum, Turbulence kinetic energy and dissipation rate was 0.5 . The solution was generally initialised based on inlet variables values. At the beginning of the solution, a noticed necessary step of starting by the simple one-equation Spallart and Allmaras model to overcome the issue of convergence instability. After around 500 iteration steps where enough steadiness level is detected, the turbulence model is switched to the adopted $\mathrm{k}-\varepsilon$ model. the convergence criteria are based on attaining a residual level of $10^{-4}$ for all flow variables. Besides that, the stability of aerodynamic coefficients is detected until variations in their values are within $\pm 1 \%$ of their obtained value. It has been noticed that all cases generally are converged after around 10000 complete iteration depending on the flow unsteadiness and geometrical complexity.

\section{RESULTS AND DISCUSSION}

The computational results obtained for TT wheel are discussed in this section as well as the validation model of ST wheel. The pressure coefficient $\mathrm{Cp}$ for any point over the wheel surface is calculated using the following equation:

$$
\mathrm{C}_{\mathrm{p}}=2\left(\mathrm{p}-\mathrm{p}_{\infty}\right) / \rho \mathrm{U}_{\infty}^{2}
$$

where $p$ is the local pressure value, $\rho$ is the air density, and $U_{\infty}$ and $p_{\infty}$ are the velocity and pressure of the upstream flow, respectively. Other dimensionless coefficients for the wheel are defined by:

$$
\begin{gathered}
C_{d}=2 F_{d} / \rho A_{f} U_{\infty}^{2} \\
C_{l}=2 F_{l} / \rho A_{f} U_{\infty}^{2}
\end{gathered}
$$




$$
C_{m}=2 T_{r} / \rho R A_{f} U_{\infty}^{2}
$$

where $F_{d}$ and $F_{l}$ are the net aerodynamic drag and lift forces applied on the wheel, respectively. $T_{r}$ is the total aerodynamic moment developed on the wheel, $\mathrm{R}$ is the wheel radius, and $A_{f}$ is the wheel frontal area.

\section{Validation Case}

In order to build a reliable computational model, it should be compared to experimental data. Amongst the previously mentioned work, Van Den Berg's [10] work was obtained using a very accurate and improved measuring system, and his results were approved in the following studies. The present work's validation case is modelled in accordance with Van Den Berg's [10] wheel geometry and experimental test conditions. The comparison was performed by considering the obtained pressure coefficient $\mathrm{Cp}$ results over the wheel surface at its middle plane (xy) as shown in Figure 8.

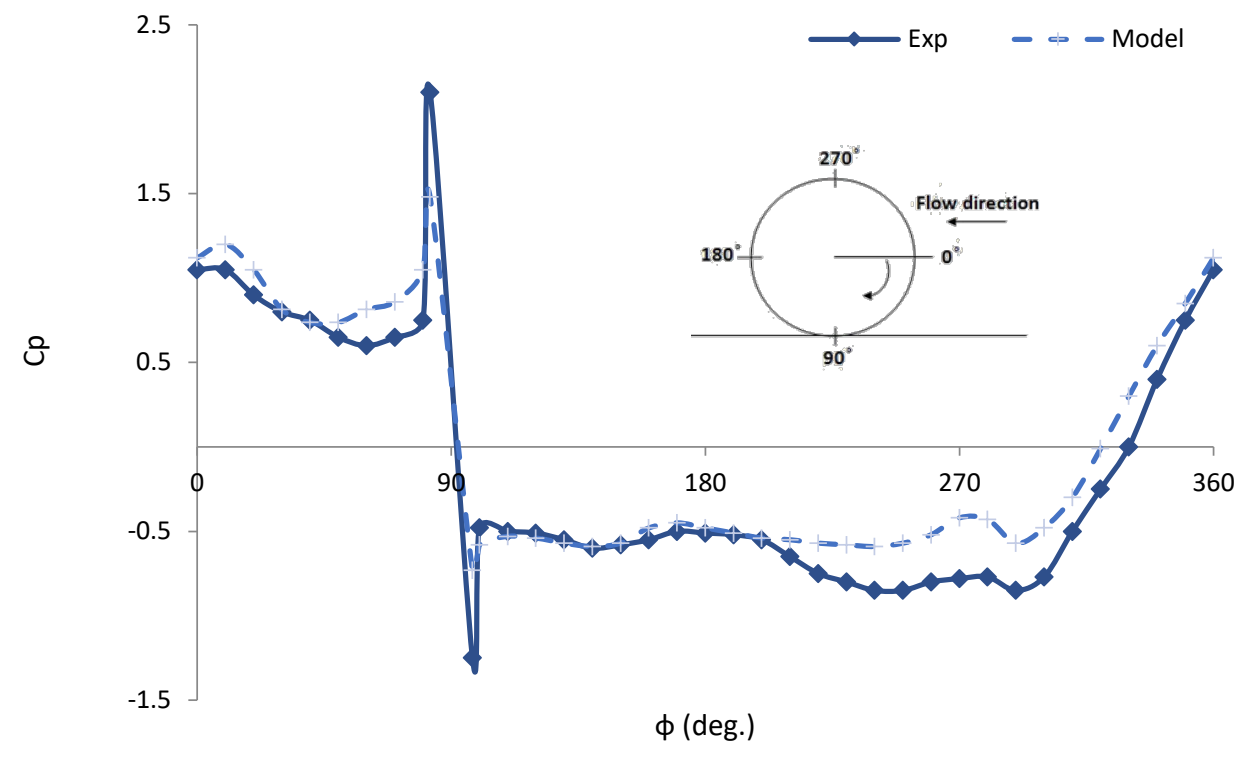

Figure 8. Comparison between the obtained model results of pressure coefficient $\mathrm{Cp}$ vs. the angle $\varphi$ (deg.) and the experimental ones done by Van Den Berg [10].

Despite the local lack in capturing the correct values of pressure and suction peaks at the contact patch region, around $25 \%$ error, the numerical results are almost matching the experimental data. Several attempts of mesh refinement were carried out in this region, and it has been observed that increasing the meshing density at this region leads to a better capturing to both peaks. The obtained values of both peaks were captured at the highest gird refinement level based on the available computational recourses. In fact, these local discrepancies are relatively acting on two points over the entire wheel circumference; hence it has a negligible effect on the aerodynamic coefficient values, which are the aims of the present work. A summary of other computed results such as drag and lift coefficients, separation and stagnation angles, and local maximum and minimum $\mathrm{Cp}$ are listed in Table 1 . These quantitative results emphasise on the previously made about the negligible effect of pressure peaks error when comparing between the experimental and the computed coefficient of drag $\mathrm{Cd}$ which shows a near-identical value and relatively accurate separation and stagnation angles except some slight differences. No experimental $\mathrm{Cl}$ value was obtained by Van Den Berg [10].

Table 1. Comparison between the computed and experimental quantitative results.

\begin{tabular}{lcccccc}
\hline Method & $\mathrm{Cd}$ & $\mathrm{Cl}$ & Separation angle $\left(^{\circ}\right)$ & Stagnation angle $\left(^{\circ}\right)$ & $\mathrm{Cp} \mathrm{min}$ & $\mathrm{Cp} \mathrm{max}$ \\
\hline Exp & 0.62 & --- & 275 & 6.5 & -1.5 & 2.1 \\
Model & 0.58 & 0.6 & 275 & 5.8 & -0.63 & 1.5 \\
\hline
\end{tabular}

\section{TT Wheel}

A direct comparison of the pressure coefficient results between the ST wheel (a) and TT wheels (b) is plotted in Figure 9 in a different representative manner to that described in the validation study. The discontinuity observed in the upper curve is due to the presence of the contact patch. The second observation is concerned with the differences in the $\mathrm{Cp}$ curve shape where it fluctuates continuously for case (b), whilst that of the case (a) is entirely smooth. This is due to the existence of the tread pads which act as a bluff body in their behaviour. Consequently, large values of $\mathrm{Cp}$ at the front of the tread pads are captured as well as low ones at their rear. Therefore, the TT wheel case shows sever local variation in the pressure coefficient curve. That emphasises the dramatically increasing moment coefficient of the TT wheel, as listed in Table 2. The negative sign in moment coefficient values indicates that the applied aerodynamic moment on the 
wheel is counter to its rotational direction. Further interesting features are related to the lobe size of Cp curve in case (b), which reveals more shrunk area than that of the case (a). That indicates lower values of drag and lift coefficients for case (b) as listed in Table 2. The decreasing in the drag and lift coefficients is due to the presence of tread pads which keep the near airflow attached to the wheel surface. This effect results in a later separation which causes a lower wake area behind the TT wheel. Figure 10 shows the computed static pressure distribution over the wheel surface at the front top region. This region is the highest influential region in the values of resistive moment where the relative velocity between the approaching accelerated flow and wheel surface is significantly high. This figure indicates that there is a pressure difference across the tread pads at the front and back areas which mainly produces a considerable value of resistive torque.

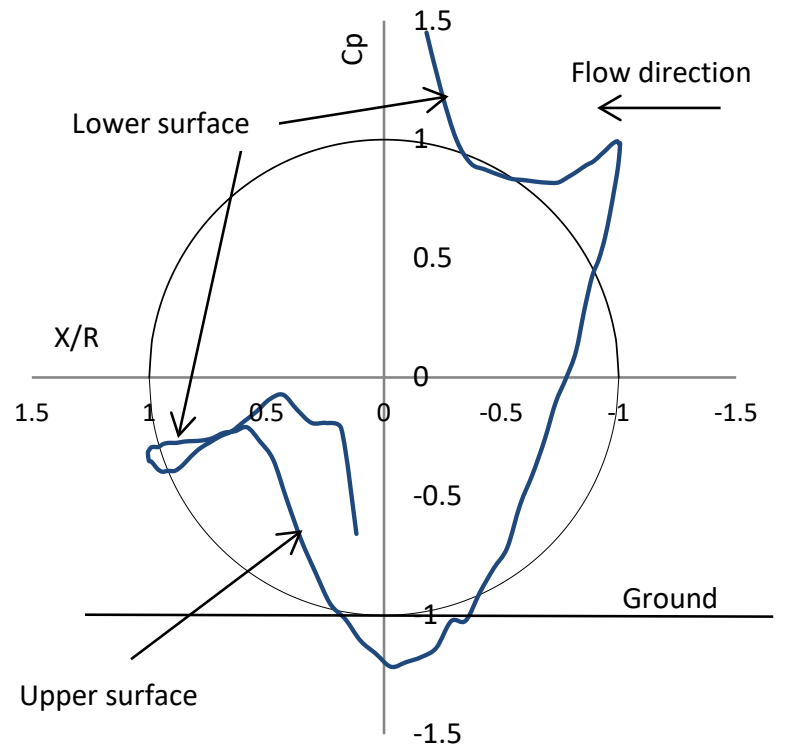

(a)

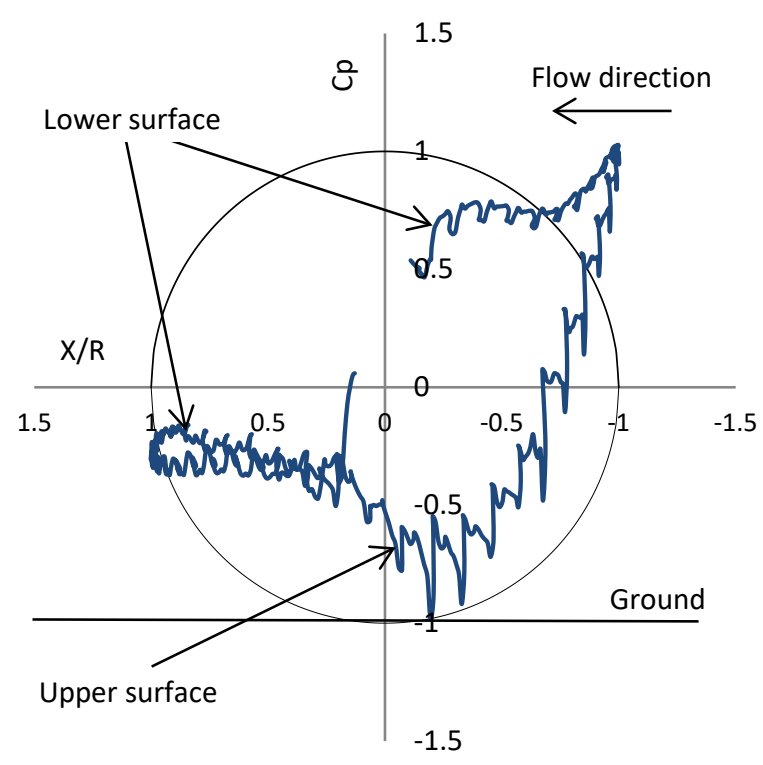

(b)

Figure 9. Distribution of the surface pressure coefficient of the two wheel cases (a) ST wheel and (b) TT wheel, at $\mathrm{h}=6 \mathrm{~mm}$

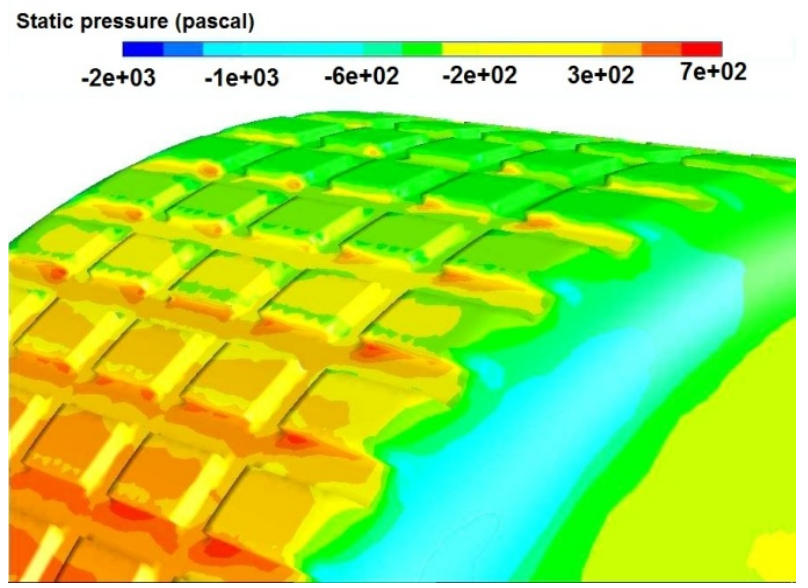

Figure 10. Surface static pressure distribution (Pascal) at the front of tread pads at the wheel front top region from $\varphi=300^{\circ}$ to $\varphi=330$ at $\mathrm{h} / \mathrm{D}=0.0097$.

Table 2. The computed aerodynamic coefficients of ST and TT wheels.

\begin{tabular}{lcc}
\hline Coef. & ST wheel & TT wheel \\
\hline $\mathrm{Cd}$ & 0.58 & 0.47 \\
$\mathrm{Cl}$ & 0.6 & 0.3 \\
$\mathrm{Cm}$ & -0.014 & -0.085 \\
\hline
\end{tabular}

\section{Effect of Tread Depth}

In this part, the effect of decreasing the tread depth is studied to minimise the frontal area of the tread elements, which in turn strongly affects the magnitude of the resistive torque. Figure 11 shows the relation between the moment coefficient and tread depth. This figure demonstrates that the moment coefficient decreases gradually by lowering the tread depth. The interesting outcome is that the decreasing trend seems to be linear except the transitional drop at h/D equal zero (ST 
wheel). No noticed changes in the coefficient of drag and lift were observed, regardless of the tread depth. This is demonstrated in Figures 11 despite the transitional rise at $\mathrm{h} / \mathrm{D}$ equal zero. This is since no significant change in the flow variables around the wheel is found from $h / D=0.0016$ to 0.0097 . This conclusion can be extracted from Figure 12, which shows the variation surface pressure for the TT wheel at different tread depths. The lobe sizes of averaged surface pressure curves have slight change for various tread depths, and this similarity in the flow characteristics around the wheel emphasises the stability in the drag and lift coefficients' outcomes. This means that the effect of later separation is gained once the ST wheel becomes TT, regardless of the depth value. Increasing the depth value just increases the quantity of incompressible flow trapped in the cavity between two tread pads.

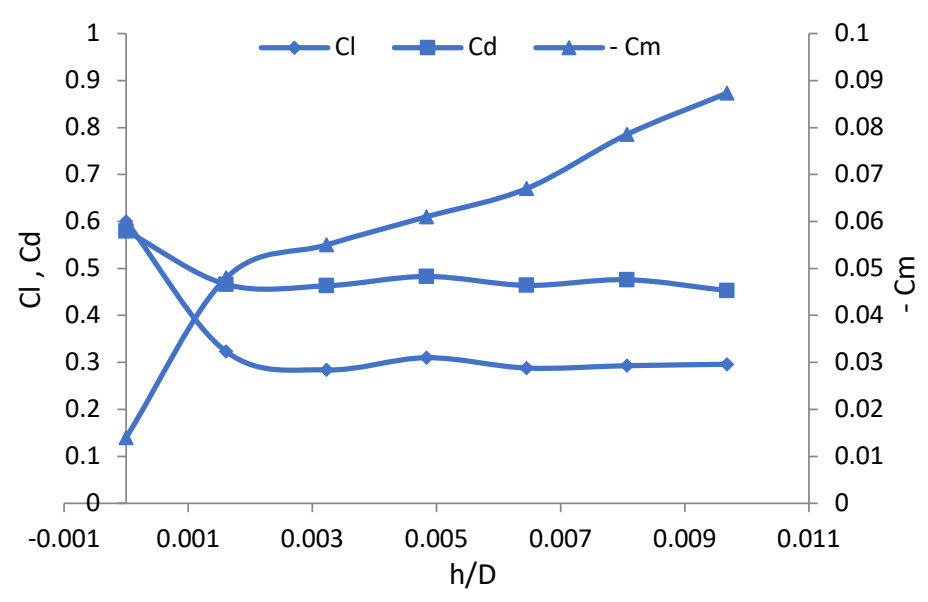

Figure 11. Variation of drag, lift, and moment coefficient with the tread depth (h) of the TT wheel model.

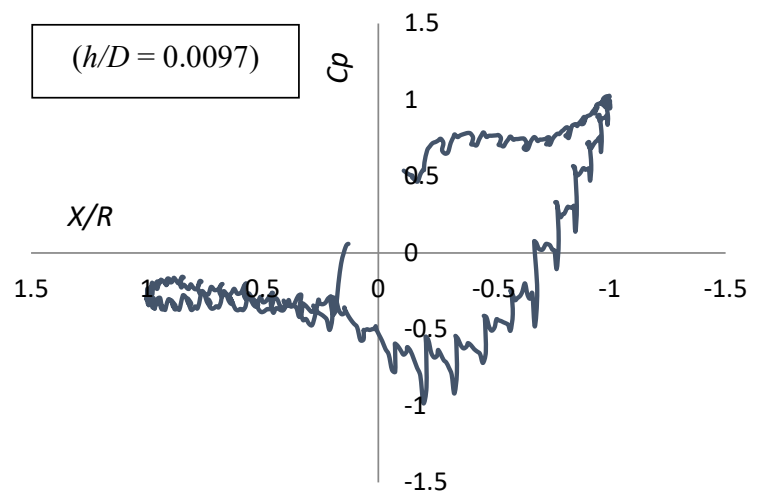

(a)

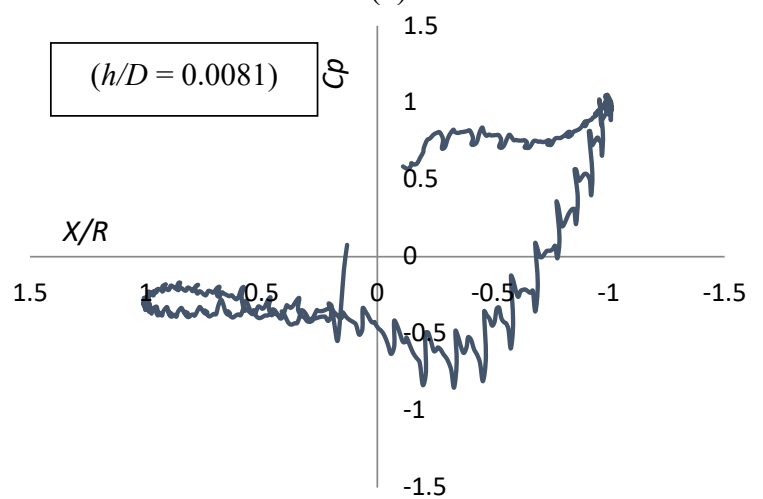

(c)

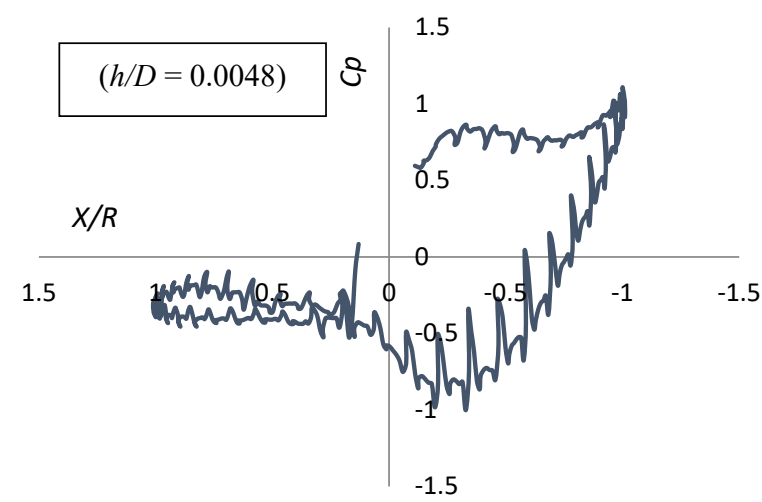

(b)

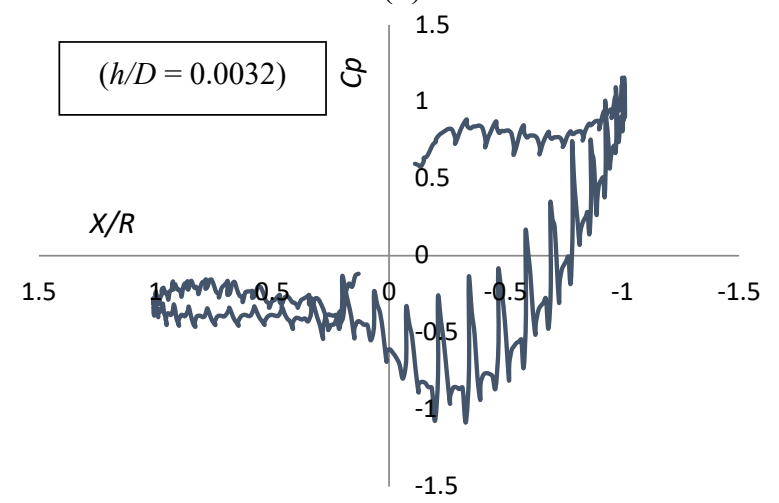

(d) 


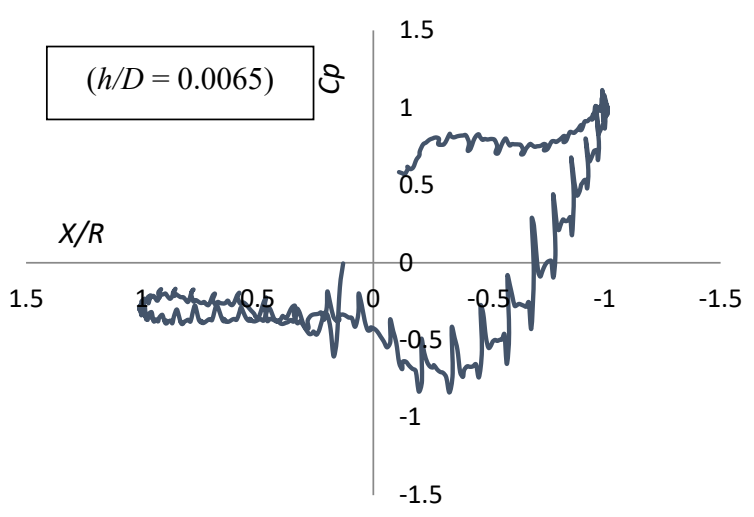

(e)

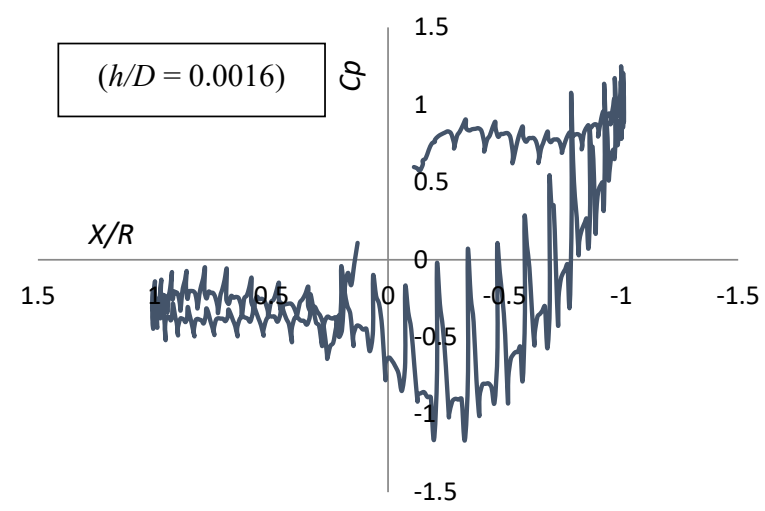

(f)

Figure 12. Variation in the surface pressure coefficient of TT wheel at different tread depths.

Another interesting observation in Figure 12 is that the local pressure variations increase as the tread depth decreases. This means that the difference between the pressure values across the tread pads increases when the tread depth decreases; however, the applied moment generally drops. In principle, the applied moment is proportionally influenced by the multiplication of the frontal pads area and the pressure differences across the tread them. However, the increase in pressure difference, the tread pads area becomes smaller and has a stronger influence over the pressure differences on the computed moment values. The reason for increased local variation in the values of $\mathrm{Cp}$ for lower tread depths is concerned with the interaction between the upcoming airflow and tread pumping effect. This pumping effect acts like a shield which prevents the wheel from being exposed to the upcoming flow. As the tread element depth becomes smaller, the pumping effect gradually diminished, and the wheel is entirely exposed to the upstream flow. This phenomenon is shown in Figure 13 and was previously suggested by Fackrell and Harvey [6], and also known as off-surface or floating separation. Fackrell argued that the induced flow acts against the approaching flow in the top region, which in turn produces strongly-effective shear stress.

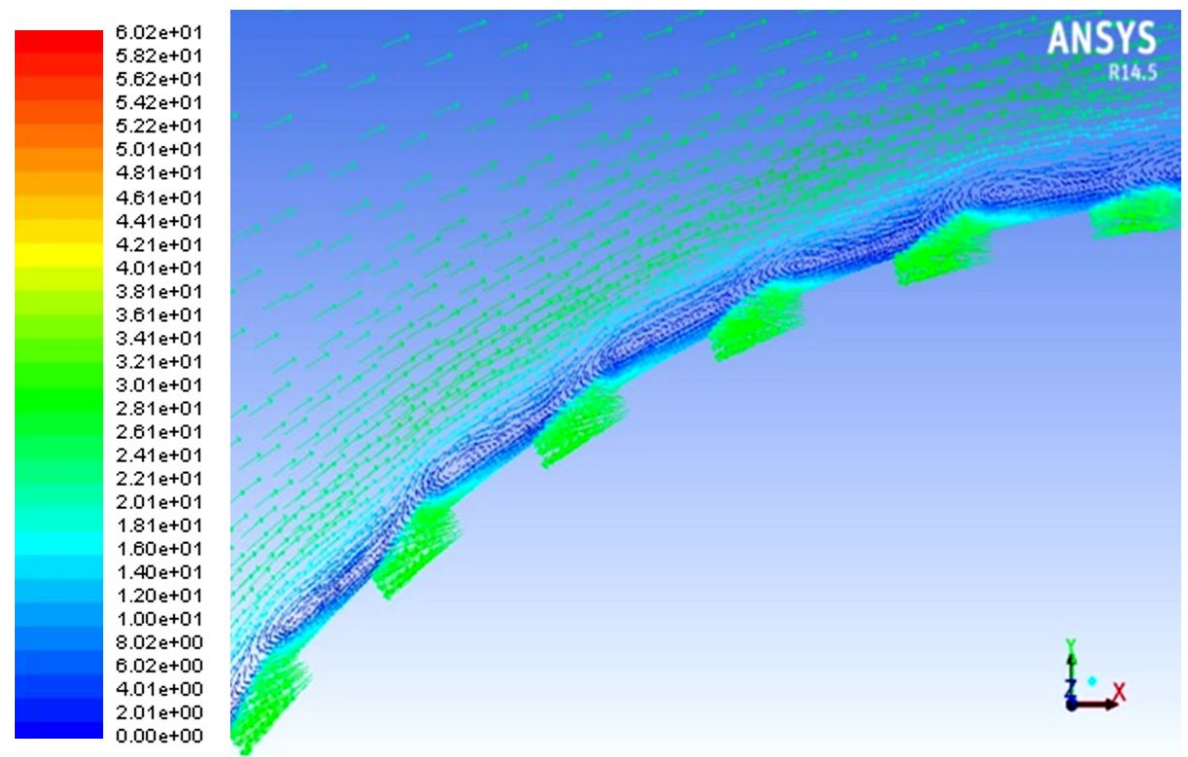

Figure 13. Normalised $\mathrm{Z}$ velocities $(\mathrm{m} / \mathrm{s})$ at the wheel middle plane $(\mathrm{xy})$ at the wheel front top region from $\varphi=300^{\circ}$ to $\varphi=330$ at $\mathrm{h} / \mathrm{D}=0.0097$

\section{CONCLUSION}

The modelling approach adopted in the present work showed good agreement to the experimental work in the verification case study as well as physically reasonable outcomes related to the TT wheel study. In addition, the realisable $\mathrm{k}-\varepsilon$ model with the standard wall function and MRF technique shows a relatively good approach to simulate the dynamic effect of the wheel rotation.

The moment coefficient of the TT wheel reaches a value of -0.085 at $6 \mathrm{~mm}$ tread depth. The effect of decreasing the tread depth can significantly decrease the moment coefficient to reach a value of -0.05 at a tread depth of $2 \mathrm{~mm}$. The decrease in tread depth does not show a noticed change in the drag and lift coefficients. The values of the resistive torque are high, especially in the TT wheel model. According to the typical Formula One rolling resistance, the resistive torque 
contributes by increasing the value of rolling moment by approximately four times. Consequently, the resistive torque has a high leverage effect on the wheel dynamic performance and is a new parameter which has to be considered.

\section{ACKNOWLEDGEMENT}

We would like to express my gratitude to the research team of CFD laboratory at Ain-shams University. We appreciate their funding, assistance for helping and aiding the advance of this research.

\section{REFERENCES}

[1] Morelli A. Aerodynamics actions on an automobile wheel. In: $1^{\text {st }}$ Symposium on Road Vehicle Aerodynamics, London, England; November, 1969.

[2] Stapleford WR, Carr GW. Aerodynamic characteristics of exposed rotating wheels. England: Lindley; 1969.

[3] Cogotti A. Aerodynamic characteristics of car wheels. In: $1^{\text {st }}$ International Conference on Impact of Aerodynamics on Vehicle Design, London, England, pp. 173-196; 1983.

[4] Fackrell JE. The aerodynamics of an isolated wheel rotating in contact with the ground. PhD thesis, University of London; 1974.

[5] Fackrell JE, Harvey JK. The aerodynamics of an isolated road wheel. In: $2^{\text {nd }}$ Symposium on Aerodynamics and Competition Automobiles, Los Angeles, California, United States, pp. 119-125; 1974.

[6] Hinson M. Measurement of the lift produced by an isolated rotating formula one wheel using a new pressure measurement system. MSc thesis, Cranfield University; 1999.

[7] Mears AP, Dominy RG, Sims-Williams DB. The Flow about an isolated rotating wheel - effects of yaw on lift, drag and flow structure. In: $4^{\text {th }}$ MIRA International Vehicle Aerodynamics Conference, Warwick, United Kingdom; October, 2002.

[8] Mears AP, Dominy RG. Racing car wheel aerodynamics - Comparisons between experimental and CFD derived flow-field data. SAE Technical Paper: 2004-01-3555; 2004.

[9] Purvis AR. The wake behind a deformable racing tire. MSc thesis, Cranfield University; 2003.

[10] Van Den Berg MA. Aerodynamic interaction of an inverted wing with a rotating wheel. PhD thesis, University of Southampton; March 2007.

[11] Knowles RD, Saddington AJ, Knowles K. On the near wake of a Formula One front wheel. Journal of Automobile Engineering 2013; 227(11):1491-502.

[12] Heyder-Bruckner J, Zhang X. The aerodynamics of an inverted wing and a rotating wheel in ground effect. PhD Thesis, University of Southampton, 2011.

[13] Sprot AJ, Sims-Williams DB, Dominy RG. The aerodynamic characteristics of a fully deformable Formula One wind tunnel tyre. SAE International Journal of Passenger Cars 2012; 5(2):1026-41.

[14] Axon L, Garry K, Howell J. An evaluation of CFD for modelling the flow around stationary and rotating wheels. SAE Technical report: $980032 ; 1998$.

[15] Skea AF, Bullen PR, Qiao J. The use of CFD to predict the air flow around a rotating wheel. In: $1^{\text {st }}$ International Conference on Vehicle Aerodynamics, United Kingdom; 1998.

[16] McManus JP. The aerodynamics of wheels in ground effect. PhD thesis, University of Southampton; 2003.

[17] WÄaschle A, Cyr S, Kuthada T, Wiedemann J. Flow around an isolated wheel -experimental and numerical comparison of two CFD codes. SAE Technical Report: 2004-01-0445; 2004.

[18] Basara B, Beader D, Przulj V. Numerical simulation of the air flow around a rotating wheel. In: $3^{\text {rd }}$ MIRA International Vehicle Aerodynamics Conference, Rugby, United Kingdom; 2000.

[19] Diasinos S, Barber TJ, Doig G. The effects of simplifications on isolated wheel aerodynamics. Journal of Wind Engineering and Industrial Aerodynamics 2015; 1(146): 90-101.

[20] Soliman MZ, Elnahas NS, Abdelaziz MA, El-Baz AR. A Computational study on the moment coefficient Cm of formula one exposed dry and wet-condition wheels. OMICS international Journal of Fluid Mech 2017; 4(149): 2476-296.

[21] Schnepf B, Schütz T, Indinger T. Further investigations on the flow around a rotating, isolated wheel with detailed tread pattern. SAE International Journal of Passenger Cars 2015;14(8): 261-74.

[22] Hobeika T, Sebben S. CFD investigation on wheel rotation modelling. Journal of Wind Engineering and Industrial Aerodynamics 2018; 1(174): 241-51.

[23] Bolzon MD, Sebben S, Broniewicz A. Effects of wheel configuration on the flow field and the drag coefficient of a passenger vehicle. International Journal of Automotive Technology 2019; 20(4): 763-77.

[24] Bridgestone Motorsport. Retrieved from http//www.bridgestonemotorsport.com; 20 March, 2015.

[25] Fluent IN. FLUENT 6.3 user's guide. Fluent documentation; 2006. 